\title{
gु \\ Spin projection and spin current density within relativistic electronic-transport calculations
}

\author{
S. Lowitzer, D. Ködderitzsch, and H. Ebert \\ Department Chemie, Physikalische Chemie, Universität München, Butenandstr. 5-13, 81377 München, Germany
}

(Received 30 July 2010; published 8 October 2010)

\begin{abstract}
A spin projection scheme is presented which allows the decomposition of the electric conductivity into two different spin channels within fully relativistic $a b$ initio transport calculations that account for the impact of spin-orbit coupling. This is demonstrated by calculations of the spin-resolved conductivity of $\mathrm{Fe}_{1-x} \mathrm{Cr}_{x}$ and $\mathrm{Co}_{1-x} \mathrm{Pt}_{x}$ disordered alloys on the basis of the corresponding Kubo-Greenwood equation implemented using the Korringa-Kohn-Rostoker coherent-potential approximation band-structure method. In addition, results for the residual resistivity of diluted Ni-based alloys are presented that are compared to theoretical and experimental ones that rely on Mott's two-current model for spin-polarized systems. The application of the scheme to deal with the spin-orbit induced spin-Hall effect is discussed in addition.
\end{abstract}

DOI: 10.1103/PhysRevB.82.140402

PACS number(s): 72.25.Ba, 71.70.Ej, 72.15.Eb, 11.80.La

During the last years research activities in spintronics increased very rapidly. The reason for the growing interest in this field is based on the close connection with fundamental scientific questions as well as its impact on technology. 1,2 Compared to standard electronics where only the charge of the electrons is used, spintronics uses the charge of the electrons in combination with the spin degree of freedom. One of the most exciting effects within spintronics is the spin-Hall effect (SHE). ${ }^{3,4}$ The SHE appears when an electric current flows through a medium with spin-orbit coupling present, leading to a spin current perpendicular to the charge current. This effect is even present in nonmagnetic materials as could be demonstrated experimentally, e.g., for Pt. ${ }^{5}$

For a theoretical investigation of effects such as the SHE it is obviously crucial to have a reliable description for the spin-dependent transport that accounts for the impact of spin-orbit coupling in a proper way. Most investigations in this field were based on the Pauli equation including spinorbit coupling explicitly as a relativistic correction term and representing the spin current density essentially by a combination of the Pauli spin matrix $\sigma_{z}$ with the conventional current density operator. ${ }^{6}$ Very few investigations have been done so far on the basis of the Dirac equation using an expression for the spin current density, albeit introduced in an ad hoc manner. ${ }^{7}$ In contrast to these approximate schemes to deal with spin-dependent transport the approach suggested by Vernes et al. ${ }^{8}$ supplies a fully relativistic and coherent description of electronic spin polarization and the associated spin current density. This approach based on the fourcomponent polarization operator $\mathcal{T}$ introduced by Bargmann and Wigner' leads, in particular, to a corresponding set of continuity equations.

In the present work we introduce spin projection operators derived from the polarization operator $\mathcal{T}$. This allows a decomposition of the conductivity into contributions from each spin channel within fully relativistic transport calculations. Applications on the spin-dependent transport of various magnetic transition-metal alloy systems demonstrate the flexibility and reliability of the approach.

Within nonrelativistic quantum mechanics the electronic spin can be described via the well-known Pauli matrices $\sigma_{i}$, specifying the nonrelativistic spin operator $\mathbf{s}=\frac{\hbar}{2} \boldsymbol{\sigma}$. Due to the fact that the Schrödinger Hamiltonian $H_{S}$ commutes with $\mathbf{s}$ the projection of the spin, e.g., to the $z$ axis, is a constant of motion. This is no longer the case within a scheme that accounts for spin-orbit coupling. The most reliable approach in this context makes use of electronic-structure calculations on the basis of the Dirac equation. It turns out that even in the simplest case of a free electron the Dirac Hamiltonian does not commute with, e.g., $s_{z}$. However, it is possible to define a generalized spin operator which commutes with the freeelectron Dirac equation and shows all characteristic properties of a spin operator. ${ }^{10,11}$

Within the fully relativistic description it is not possible to decompose the conductivity in a strict sense into spin-up and spin-down contributions in a simple way. Therefore, one may use approximative schemes or one can switch to scalarrelativistic calculations ${ }^{12,13}$ to decompose the conductivity into two different spin channels. The disadvantage of these two approaches is that approximative schemes work only under certain circumstances and scalar-relativistic calculations neglect all scattering events that lead to a spin flip due to the fact that such calculations neglect spin-orbit coupling. To avoid such shortcomings a proper relativistic spin projection operator is necessary.

The starting point of our derivation of suitable relativistic spin projection operators is based on the four-vector polarization operator $\mathcal{T}$ which was derived by Bargmann and Wigner ${ }^{9}$

$$
\begin{gathered}
\mathbf{T}=\beta \mathbf{\Sigma}-\frac{\gamma_{5} \boldsymbol{\Pi}}{m c}, \\
T_{4}=i \frac{\boldsymbol{\Sigma} \cdot \boldsymbol{\Pi}}{m c}
\end{gathered}
$$

with the kinetic momentum $\boldsymbol{\Pi}=\left(\hat{\mathbf{p}}+\frac{|e|}{c} \mathbf{A}\right) \rrbracket_{4}$ and the canonical momentum $\hat{\mathbf{p}}$. The matrices $\boldsymbol{\Sigma}$ are the relativistic Pauli matrices, $\beta$ is one of the standard Dirac matrices, and ${ }^{11}$ 


$$
\gamma_{5}=\left(\begin{array}{cc}
0 & -1_{2} \\
-1_{2} & 0
\end{array}\right) .
$$

The operator $\mathcal{T}$ can be considered as a generalized spin operator which commutes with the field-free Dirac Hamiltonian ${ }^{11}$

$$
H^{\mathrm{free}}=c \boldsymbol{\alpha} \cdot \hat{\mathbf{p}}+\beta m c^{2} .
$$

In addition, the components $\mathcal{T}_{\mu}$ are the generators of the little group that is a subgroup of the group of Lorentz transformations. ${ }^{10}$ In comparison to other suggested forms of polarization operators the operator $\mathcal{T}$ is gauge invariant ${ }^{11}$ and therefore the appropriate basis for calculations which include electromagnetic fields.

A widely used relativistic scheme to deal with magnetic solids within spin density-functional theory was introduced by MacDonald and Vosko. ${ }^{14}$ The corresponding Dirac Hamiltonian

$$
H=c \boldsymbol{\alpha} \cdot \hat{\mathbf{p}}+\beta m c^{2}+V_{\mathrm{eff}}+\beta \boldsymbol{\Sigma} \cdot \mathbf{B}_{\mathrm{eff}},
$$

includes an effective scalar potential $V_{\text {eff }}$ and an effective magnetic field $\mathbf{B}_{\text {eff }}$ coupling only to the spin degree of freedom. For the subsequent discussion we choose $\mathbf{B}_{\text {eff }}=B(r) \hat{\mathbf{e}}_{z}$ as frequently done within electronic-structure calculations. The commutator of $\mathcal{T}$ and $H$ is nonzero which shows that $\mathcal{T}$ is no longer a constant of motion.

From $\mathcal{T}$ corresponding spin projection operators $\mathcal{P}^{ \pm}$can be derived by demanding

$$
\begin{aligned}
& \mathcal{P}^{+}+\mathcal{P}^{-}=1, \\
& \mathcal{P}^{+}-\mathcal{P}^{-}=\mathcal{T},
\end{aligned}
$$

or equivalently

$$
\mathcal{P}^{ \pm}=\frac{1}{2}(1 \pm \mathcal{T})
$$

Focusing on the spatial $z$ component leads to the following expression:

$$
T_{3}=\beta \Sigma_{z}-\frac{\gamma_{5} \Pi_{z}}{m c} .
$$

Making use of the relation $\mathbf{B}=\nabla \times \mathbf{A}$ between the vector potential $\mathbf{A}$ and the magnetic field $\mathbf{B}, \mathbf{A}$ has only nonzero components in the $x y$ plane if $\mathbf{B} \| \hat{\mathbf{e}}_{z}$ [see Eq. (5)], i.e., $A_{z}=0$. For the spin projection operators this leads to

$$
\mathcal{P}_{z}^{ \pm}=\frac{1}{2}\left[1 \pm\left(\beta \Sigma_{z}-\frac{\gamma_{5} \hat{p}_{z}}{m c}\right)\right] .
$$

Inspired by Vernes et al. ${ }^{8}$ where, using $\mathcal{T}$, an explicit expression for a polarization current density operator is given we use the above derived spin-projection scheme involving $\mathcal{T}$ and combine it with the former to obtain a spin-projected current density operator involving the conventional relativistic electron current density operator

$$
\hat{j}_{\mu}=-|e| c \boldsymbol{\alpha}_{\mu},
$$

where $\boldsymbol{\alpha}_{\mu}$ is one of the standard Dirac matrices. ${ }^{11}$ Accordingly, we get an operator for the spin-projected current density by combining $\mathcal{P}_{z}^{ \pm}$and $\hat{j}_{\mu}$ which leads to $\mathcal{J}_{\mu}^{ \pm}=\mathcal{P}_{z}^{ \pm} \hat{j}_{\mu}$.

Using $\mathcal{J}_{\mu}^{ \pm \pm}$to represent the observable within Kubo's linear-response formalism one can derive expressions for a corresponding spin-projected conductivity tensor (the details will be published elsewhere). Restricting to the symmetric part of the tensor one arrives at

$$
\sigma_{\mu \nu}^{z \pm}=\frac{\hbar}{\pi N \Omega} \operatorname{Tr}\left\langle\mathcal{J}_{\mu}^{z \pm} \Im G^{+}\left(E_{F}\right) \hat{j}_{\nu} \Im G^{+}\left(E_{F}\right)\right\rangle .
$$

Here $N$ is the number of atomic sites, $\Omega$ the volume per atom, $\hat{j}_{\mu}$ is the current density operator $(\mu=x, y, z)$, and $\Im G^{+}\left(E_{F}\right)$ is the imaginary part of the retarded one-particle Green's function at the Fermi energy $E_{F}$.

Equation (12) is obviously the counterpart to the conventional Kubo-Greenwood equation ${ }^{15}$ for the spin-integrated conductivity that is recovered by replacing $\mathcal{J}_{\mu}^{ \pm}$by $\hat{j}_{\mu}$.

For the determination of $\Im G^{+}\left(E_{F}\right)$ we use multiplescattering theory (MST) which is the basis of the KKR bandstructure method. Within MST the real-space representation of $\Im G^{+}$has the following form: ${ }^{16}$

$$
\Im G^{+}\left(\mathbf{r}, \mathbf{r}^{\prime}, E\right)=\Im \sum_{\Lambda_{1} \Lambda_{2}} Z_{\Lambda_{1}}^{n}\left(\mathbf{r}_{n}, E\right) \tau_{\Lambda_{1} \Lambda_{2}}^{n m}(E) Z_{\Lambda_{2}}^{m \times}\left(\mathbf{r}_{m}, E\right)
$$

with $\mathbf{r}=\mathbf{R}_{n}+\mathbf{r}_{n}$ and $\mathbf{r}^{\prime}=\mathbf{R}_{m}+\mathbf{r}_{m}$. Using a fully relativistic implementation, the wave functions $Z_{\Lambda}^{n}\left(Z_{\Lambda}^{n \times}\right)$ are the regular right (left) hand side solutions of the Dirac equation within cell $n, \tau_{\Lambda \Lambda^{\prime}}^{n m}$ is the scattering path operator and $\Lambda=(\kappa, \mu)$ with $\kappa$ and $\mu$ being the relativistic spin-orbit and magnetic quantum numbers. ${ }^{11}$

The configurational average for a disordered alloyindicated by the brackets $\langle\cdots\rangle$ in Eq. (12) - is taken by means of the coherent-potential approximation (CPA). ${ }^{15}$ The scheme outlined above has been implemented by a corresponding extension of the formalism worked out by Butler ${ }^{15}$ to calculate the residual resistivity of disordered alloys on the basis of the Kubo-Greenwood equation. As a first step, the underlying electronic structure of the investigated disordered alloy systems has been calculated self-consistently on the basis of local spin-density approximation (LSDA) using the parametrization of Vosko et al. ${ }^{17}$ For the band-structure calculations the fully relativistic version of the Korringa-KohnRostoker (KKR) method ${ }^{18}$ has been used in combination with the CPA alloy theory to account for chemical disorder. The CPA has been exploited, in particular, to perform the configurational average when calculating the conductivity tensor elements and when dealing with the associated vertex corrections. ${ }^{15}$ These calculations have been done using a cutoff for the angular momentum expansion at $l_{\max }=3$ to ensure convergence for the investigated transition-metal systems.

As a first application of the presented projection scheme the spin-resolved conductivity of the alloy system $\mathrm{Fe}_{1-x} \mathrm{Cr}_{x}$ has been calculated assuming the magnetization to be 

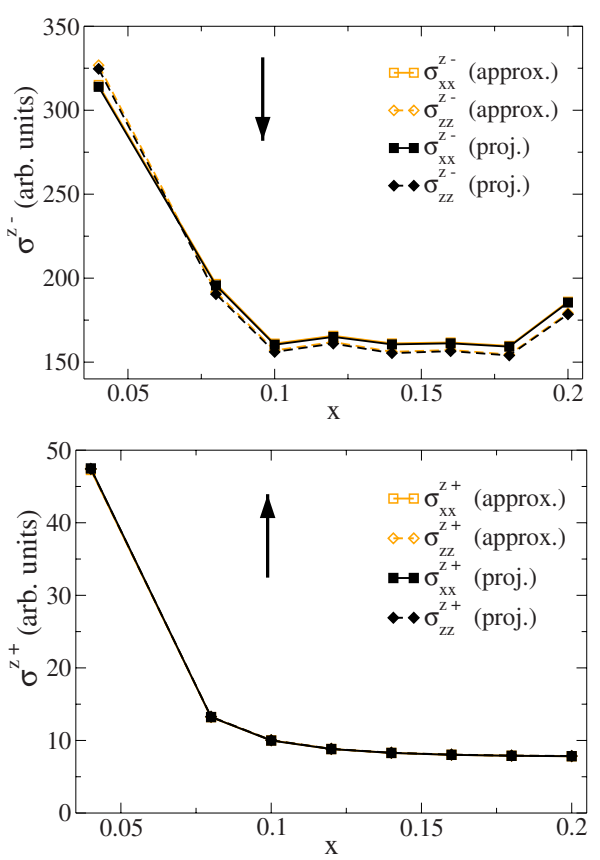

FIG. 1. (Color online) Spin-resolved conductivity tensor elements $\sigma_{x x}^{z+(-)}$ and $\sigma_{z z}^{z+(-)}$ of $\mathrm{Fe}_{1-x} \mathrm{Cr}_{x}$ calculated for the magnetization pointing along the $z$ axis (full symbols). In addition, results are shown that have been obtained using an approximate scheme (open symbols) (Ref. 19).

aligned along the $z$ axis. The presence of the spin-orbit coupling gives rise to the anomalous magnetoresistance (AMR) with the conductivity tensor elements $\sigma_{x x}=\sigma_{y y} \neq \sigma_{z z}$ for this situation. The reduced symmetry is also reflected by the spinprojected conductivities $\sigma_{x x}^{z+(-)}$ and $\sigma_{z z}^{z+(-)}$, as can be seen in Fig. 1. Obviously, the conductivity is quite different for the two spin channels. This behavior can be traced back straightforwardly to the electronic structure of the alloy system around the Fermi energy that can be represented in a most detailed way in terms of the spin-projected Bloch spectral function (BSF).$^{20}$ While for the spin-down subsystem there exists a well-defined Fermi surface with dominant $s p$ character corresponding to a sharp BSF, the spin-up subsystem is primarily of $d$ character that is much more influenced by the chemical disorder in the system leading to a BSF with rather washed-out features. ${ }^{20}$ As the width of the BSF can be seen as a measure for the inverse of the electronic lifetime the very different width found for the two spin subsystems explain the very different spin-projected conductivities.

Figure 1 shows in addition results that have been obtained on the basis of an approximate spin-projection scheme that was suggested recently. ${ }^{19}$ Within this scheme the matrices occurring in the Kubo-Greenwood equation for the conductivity are transformed from the standard relativistic representation [using the quantum numbers $\Lambda=(\kappa, \mu)$ as labels] to a spin-projected one [using the quantum numbers $L$ $=\left(l, m_{l}, m_{s}\right)$ as labels]. Suppressing the spin-flip term of the current density-matrix elements $J_{L L^{\prime}}$ one can easily split the conductivity into spin-up and spin-down contributions and an additional spin-flip contribution $\sigma^{z+-}$ that is related to the spin-off-diagonal elements of the scattering path operator $\tau$. For $3 d$ elements with a relatively low spin-orbit coupling it

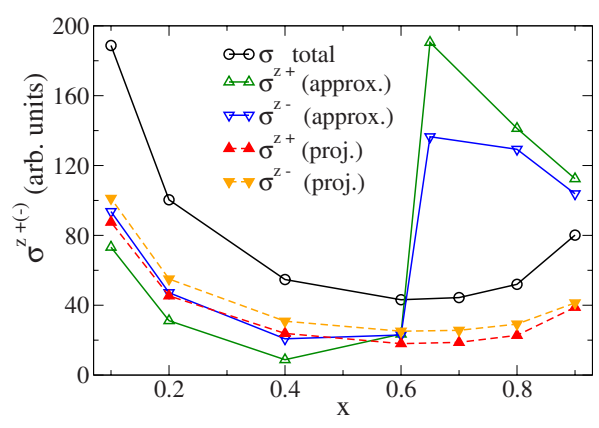

FIG. 2. (Color online) Isotropic spin-resolved conductivity $\sigma^{z+(-)}=\left(2 \sigma_{x x}^{z+(-)}+\sigma_{z z}^{z+(-)}\right) / 3$ of $\mathrm{Co}_{1-x} \mathrm{Pt}_{x}$ for the magnetization pointing along the $z$ axis (full symbols). In addition, results are shown that have been obtained using an approximate scheme (open symbols) (Ref. 19).

was found that the neglect of spin-off-diagonal elements of $J_{L L^{\prime}}$ is well justified and that $\sigma^{z+}$ is quite small. In fact the spin-projected conductivities $\sigma_{x x}^{z+(-)}$ and $\sigma_{z z}^{z+(-)}$ obtained by the approximate scheme compare very well with the results based on the scheme presented here (see Fig. 1).

In order to demonstrate the limitations of the approximate scheme from Ref. 19 the isotropic spin-resolved conductivity for $\mathrm{Co}_{1-x} \mathrm{Pt}_{x}$ is shown in Fig. 2. It turns out, that the approximative scheme fails especially for high Pt concentrations. This can be attributed to an increased spin-orbit interaction for which the assumptions on which this scheme is based are no longer fulfilled.
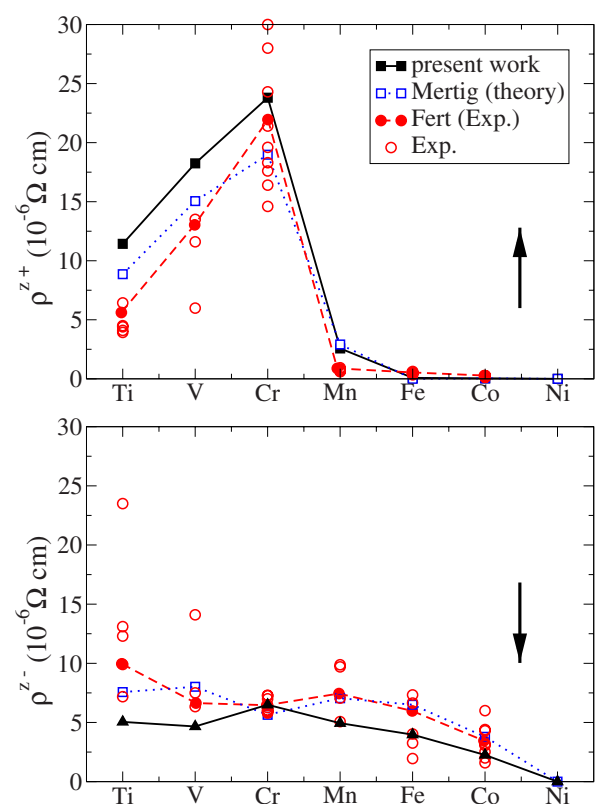

FIG. 3. (Color online) Isotropic spin-resolved resistivity of $\mathrm{Ni}$ with $3 d$ transition-metal impurities $(1 \%)$ obtained by the present scheme (full squares) compared to theoretical data from Mertig et al. (Ref. 21) (blue squares/dotted line), experimental data from Fert (Ref. 22) (full red circles/dashed line), and other experimental data (see Ref. 23, open red circles). For the deduction of the spinresolved resistivity from experimental data see the note in the text. The top and the bottom panel show the data for spin up and for spin down, respectively. 
As another application of the presented scheme results for diluted Ni-based alloys with $x_{\mathrm{Ni}}=0.99$ are shown in Fig. 3 in terms of the isotropic residual resistivities $\rho^{z+(-)}=\left[\left(2 \sigma_{x x}^{z+(-)}\right.\right.$ $\left.\left.+\sigma_{z z}^{z+(-)}\right) / 3\right]^{-1}$. As one notes, the resistivity for the two spin channels show a rather different variation with the atomic number of the impurities. This can be traced back again to the spin-projected electronic structure of $\mathrm{Ni}$ at the Fermi level and the position of the impurity $d$ states. ${ }^{21}$ In Fig. 3 the results of spin-polarized calculations by Mertig et al. ${ }^{21}$ have been added, that were done in a scalar-relativistic way, i.e., ignoring spin-orbit coupling, on the basis of the Boltzmann formalism and by making use of the two-current model. In spite of the various differences between this approach and the presented scheme, the resulting spin-projected resistivities agree fairly well. This also holds concerning corresponding experimental data that have been deduced from measurements relying on the two-current model.

In summary, a scheme for a spin projection within transport calculations on the basis of the Kubo formalism has been presented. The applications presented were restricted to the diagonal elements of the corresponding conductivity ten- sor described by a Kubo-Greenwood-type equation. Results obtained for the disordered alloy systems $\mathrm{Fe}_{1-x} \mathrm{Cr}_{x}, \mathrm{Co}_{1-x} \mathrm{Pt}_{x}$, and diluted Ni-based alloys were compared to results based on an alternative but approximate projection scheme and theoretical as well as experimental data based on the twocurrent model. The good agreement found for the investigated systems ensures the consistency and reliability of the presented scheme. Accordingly, this is expected to hold also when dealing with spin-projected off-diagonal conductivities as, e.g., $\sigma_{x y}^{z+(-)}$ on the basis of Kubo-Středa-type equations. This will give access, in particular, to the spin-projected Hall conductivity in magnetic materials as well as to the spin-Hall conductivity in nonmagnetic materials. Work along this line is in progress.

The authors would like to thank the DFG for financial support within the SFB 689 "Spinphänomene in reduzierten Dimensionen." D.K. in addition acknowledges support from the DFG priority program SPP 1145 "Modern and universal first-principles methods for many-electron systems in chemistry and physics."
${ }^{1}$ S. A. Wolf, D. D. Awschalom, R. A. Buhrman, J. M. Daughton, S. von Molnár, M. L. Roukes, A. Y. Chtchelkanova, and D. M. Treger, Science 294, 1488 (2001).

${ }^{2}$ D. Awschalom and N. Samarth, Phys. 2, 50 (2009).

${ }^{3}$ J. Sinova, D. Culcer, Q. Niu, N. A. Sinitsyn, T. Jungwirth, and A. H. MacDonald, Phys. Rev. Lett. 92, 126603 (2004).

${ }^{4}$ S. O. Valenzuela and M. Tinkham, Nature (London) 442, 176 (2006).

${ }^{5}$ T. Kimura, Y. Otani, T. Sato, S. Takahashi, and S. Maekawa, Phys. Rev. Lett. 98, 156601 (2007).

${ }^{6}$ T. Tanaka, H. Kontani, M. Naito, T. Naito, D. S. Hirashima, K. Yamada, and J. Inoue, Phys. Rev. B 77, 165117 (2008).

${ }^{7}$ G. Y. Guo, Y. Yao, and Q. Niu, Phys. Rev. Lett. 94, 226601 (2005).

${ }^{8}$ A. Vernes, B. L. Györffy, and P. Weinberger, Phys. Rev. B 76, 012408 (2007).

${ }^{9}$ V. Bargmann and E. P. Wigner, Proc. Natl. Acad. Sci. U.S.A. 34, 211 (1948).

${ }^{10}$ D. M. Fradkin and R. H. Good, Rev. Mod. Phys. 33, 343 (1961).

${ }^{11}$ M. E. Rose, Relativistic Electron Theory (Wiley, New York, 1961).
${ }^{12}$ H. Ebert, A. Vernes, and J. Banhart, Phys. Rev. B 54, 8479 (1996).

${ }^{13}$ J. Banhart, H. Ebert, and A. Vernes, Phys. Rev. B 56, 10165 (1997).

${ }^{14}$ A. H. MacDonald and S. H. Vosko, J. Phys. C 12, 2977 (1979).

${ }^{15}$ W. H. Butler, Phys. Rev. B 31, 3260 (1985).

${ }^{16}$ J. S. Faulkner and G. M. Stocks, Phys. Rev. B 21, 3222 (1980).

${ }^{17}$ S. H. Vosko, L. Wilk, and M. Nusair, Can. J. Phys. 58, 1200 (1980).

${ }^{18} \mathrm{H}$. Ebert, in Electronic Structure and Physical Properties of Sol$i d s$, Lecture Notes in Physics Vol. 535, edited by H. Dreyssé (Springer, Berlin, 2000), p. 191.

${ }^{19}$ V. Popescu, H. Ebert, N. Papanikolaou, R. Zeller, and P. H. Dederichs, J. Phys.: Condens. Matter 16, S5579 (2004).

${ }^{20}$ S. Lowitzer, D. Ködderitzsch, H. Ebert, and J. B. Staunton, Phys. Rev. B 79, 115109 (2009).

${ }^{21}$ I. Mertig, R. Zeller, and P. H. Dederichs, Phys. Rev. B 47, 16178 (1993).

${ }^{22}$ A. Fert, Rev. Mod. Phys. 80, 1517 (2008).

${ }^{23}$ J. W. F. Dorleijn and A. R. Miedema, J. Phys. F: Met. Phys. 5, 487 (1975). 